\title{
Deterioro de la salud mental asociada a la violencia de pareja: Diferente impacto en la psicopatología de las mujeres
}

\section{The mental health damage of the intimate partner violence: Different impact on the abused women psychopathology}

Fecha de recepción: 19-07-2017

Fecha de aceptación: 7-11-2017
$M^{a}$ Francisca Savall Rodríguez

Dra. Psicología. Psicóloga del Instituto de Medicina Legal y Ciencias

Forenses de Valencia

Concepción Blasco Ros

Dra. Psicobiología. Profesora Asociada de la Facultad de Psicología de Valencia.

Manuela Martínez Ortíz

Catedrática de Psicobiología. Departamento de Psicobiología de la Facultad de Psicología de Valencia

\section{resumen/alstract:}

Generalmente, los estudios realizados sobre víctimas de violencia de pareja (VP) se han centrado en investigar el impacto de dicha violencia sobre la salud de las mujeres y, más concretamente, sobre su salud mental, obteniéndose que las víctimas presentan un peor estado de salud frente a las no víctimas. Consecuentemente, el daño psíquico encontrado ha sido utilizado también como un medidor de la gravedad de la violencia sufrida. El objetivo principal de este trabajo fue comprobar si las mujeres presentaban distintos perfiles de deterioro en su salud mental en función del tipo de violencia sufrida. Se llevó a cabo un estudio transversal con 108 mujeres víctimas de VP que fueron divididas en tres grupos: sin síntomas ( $n=30)$, con depresión $(n=50)$, con depresión y Trastorno por Estrés Postraumático (TEPT) (n=28). Los análisis estadísticos realizados establecieron que el desarrollo de depresión, depresión/TEPT o el no padecer síntomas de estos trastornos no se asociaba al tipo de violencia sufrida (física, psicológica y/o sexual) al aportar datos estadísticamente no significativos. Estos resultados indican que las mujeres víctimas no conforman un grupo homogéneo entre ellas, apuntando que no debiera utilizarse únicamente el deterioro presentado en su salud mental como la variable que valore la gravedad de la violencia sufrida.

In general, the studies carried out on victims of intimate partner violence (IPV) have focused on research the impact of that violence on the abused women's health, in particular, on their mental health, concluding that abused women are much more likely than nonvictims to have poor health. Therefore, the psychological damage has been used as a severity measure of the violence suffered. The main purpose of this work was determined if women experienced different degrees of mental health impairment according to the type of violence suffered. The cross-sectional study followed 108 victims of IPV distributed into three groups: women without symptoms ( $n=30)$, women suffering depression ( $n=50)$ and women suffering depression and posttraumatic stress disorder (PTSD) ( $n=28)$. The statistical analysis carried out determined the development of depression, depression/PTSD or not suffering any evidence of these symptoms are not associated with the type of violence (physical, psychological and/or sexual) providing non-significant statistical data. These results showcase abused women are not a homogeneous group, pointing out a deterioration of abused women's mental health should not be used as a variable which determine the severity of the violence suffered.

\section{palabras clave/keywords:}

Violencia de pareja, depresión, trastorno por estrés postraumático, daño psíquico, salud mental, víctima.

Intimate partner violence, depression, posttraumatic stress disorder, psychological damage, mental health, abused women. 


\section{Introducción}

Según la Organización Mundial de la Salud (OMS, 2006), la violencia de pareja se define como el comportamiento de la pareja o ex pareja que causa daño físico, sexual o psicológico, incluidas la agresión física, la coacción sexual, el maltrato psicológico y las conductas de control.

Una de las principales características de la violencia de pareja es la sensación de riesgo constante y de pérdida de control que viven las mujeres víctimas, aun cuando no está ocurriendo el evento traumático (Kaysen, Resick y Wise, 2003). Por tanto, estas situaciones suelen derivar en estresores de tipo crónico que conllevan un profundo desgaste sobre las mujeres (Koolhaas et al., 2011) y ejercen un notable efecto perjudicial sobre su salud física y mental (Campbell, 2002; Dutton, Green, Kaltman, Roesch, Zeffiro y Krause, 2006).

En relación con la salud mental, la depresión es el trastorno más claramente relacionado con haber sido víctima de violencia de pareja (Chuang Cattoi, McCall-Hosenfeld, Camacho, Dyer, y Weisman, 2012; Craparo, Gori, Petruccelli, Cannella y Simonelli, 2014; Devries et al., 2013; Dougé, Lehman y McCall-Hosenfeld, 2014; Torres et al., 2013), seguido del Trastorno por Estrés Postraumático (TEPT) (Hansen, Eriksen, y Elklit, 2014; Hellmuth, Jaquier, Swan y Sullivan, 2014; Kelly y Pich, 2014; Lilly, Howell y Graham-Bermann, 2014), estimándose una prevalencia de este trastorno que oscila entre el 40 y el $60 \%$ en este tipo de víctimas (Dutton et al., 2006; Sarasua, Zubizarreta, Echeburúa y de Corral, 2007).

La cronicidad con que se produce este tipo de situación estresante puede explicar la alta prevalencia de depresión en las mujeres víctimas de violencia de pareja, ya que el mantenimiento del estrés resulta en un exceso en los niveles de glucocorticoides (cortisol), los cuales provocan cambios bioquímicos y una desregulación a nivel hormonal que repercute sobre los sistemas endocrino e inmune propiciando el desarrollo de enfermedades (Heim et al., 2000; McEwen, 2004), caracterizándose precisamente la depresión crónica por la hipercortisolemia (Lok et al., 2012), es decir, por una presencia de niveles elevados y sostenidos de cortisol (Hardeveld et al., 2014). Por el contrario, el desarrollo del TEPT aparece más relacionado con la respuesta psicobiológica y psicológica que se emite tras la vivencia del trauma (Ehring, Ehlers, Cleare y Glucksman, 2008).

En las investigaciones realizadas sobre víctimas de violencia de pareja, los grupos de estudio suelen establecerse para efectuar comparaciones entre las mujeres víctimas frente a las no víctimas, hallándose inequívocamente una mayor incidencia de depresión y TEPT en las mujeres que han sido víctimas de cualquier tipo de violencia. Sin embargo, cuando las mujeres víctimas son subdivididas a su vez en función del tipo de violencia experimentada (física, psicológica, sexual), suele encontrarse que la violencia sexual se relaciona con una mayor gravedad de la sintomatología presentada (Benice, Resick, Mechanic y Astin, 2003; Honda et al. 2017).

Otra línea de investigación más reciente es la que efectúa sus grupos de trabajo en función de la presencia de sintomatología depresiva o de TEPT (Griffin, Resick y Yehuda, 2005; Johnson, Delahanty y Pinna, 2008; Patró-Hernández, Corbalán y Limiñana, 2007; Pérez y Johnson, 2008), estableciendo su objetivo de trabajo en comprobar el efecto de estos tras- 
tornos sobre otras variables relacionadas con la situación de violencia, tales como la gravedad de la violencia, los estilos de personalidad asociados o el funcionamiento del sistema endocrino de las mujeres.

En el ámbito jurídico, la reciente promulgación de la Ley 4/2015 de 27 de abril, del Estatuto de la Víctima del Delito establece un concepto de víctima omnicomprensivo que se extiende a toda persona que haya sufrido un perjuicio físico, moral o económico como consecuencia de un delito. Desde la psicología forense, el daño psíquico queda definido como la medida de los efectos de un acto delictivo en la salud mental o sufrimiento emocional (Arce y Fariña, 2009) y haría alusión a todos aquellos desajustes psicológicos derivados de la exposición de la persona a una situación de victimización (Muñoz, 2013).

En estas definiciones subyacen dos factores como son la causación y las consecuencias, es decir, el delito y las secuelas, lo cual aplicado al tema que nos ocupa vendría a significar que las características de la violencia de pareja sufrida por las mujeres (tipo, duración, frecuencia, gravedad) estarían en interrelación con la gravedad de las secuelas manifestadas por las víctimas.

Llegados a este punto, el objetivo del presente trabajo ha sido comprobar si las mujeres víctimas presentan diferentes perfiles de deterioro en su salud mental y relacionar dichos perfiles con el tipo de violencia que han sufrido. Se espera encontrar diferencias en los perfiles de salud mental en función del tipo de violencia del que han sido víctimas las mujeres y que las mujeres que han sufrido más de un tipo de violencia presenten un perfil de deterioro más grave en su salud mental.

\section{Método}

\section{Participantes}

108 mujeres víctimas de violencia de pareja participaron en este trabajo, las cuales han sido distribuidas en tres grupos en función de la presencia/ausencia de sintomatología depresiva y su posible comorbilidad con un TEPT, quedando los grupos establecidos como sigue: 1) Grupo de violencia de pareja (VP) sin síntomas, mujeres víctimas de violencia de pareja que no presentan síntomas de depresión ni cumplen criterios diagnósticos de TEPT $(\mathrm{n}=30), 2)$ Grupo VP con depresión, mujeres víctimas de violencia de pareja que presentan síntomas de depresión pero no cumplen criterios diagnósticos de TEPT (n=50) y 3) Grupo VP con depresión/TEPT, mujeres víctimas de violencia de pareja que presentan síntomas de depresión y además cumplen criterios diagnósticos de TEPT $(n=28)$.

\section{Procedimiento}

La recogida de datos se realizó a través de la cumplimentación de una entrevista estructurada por parte de un grupo de psicólogas y el pase de cuestionarios estandarizados. Las variables evaluadas fueron las siguientes: edad, nivel de educación, estado civil, depresión, TEPT y tipo de violencia sufrida: física, psicológica, sexual.

Las mujeres víctimas de violencia de pareja fueron contactadas a través de los Centro Mujer 24 horas de la Comunidad Valenciana, situados en las tres capitales de provincia (Alicante, 
Castellón y Valencia). Todas las mujeres que decidieron participar, rellenaron y firmaron un consentimiento informado. Cada sesión tuvo una duración media de 1 h y 30 minutos.

\section{Instrumentos}

El cuestionario mediante el que se evaluaron los síntomas de depresión en las mujeres fue la versión de 21 ítems del Inventario de Depresión de Beck (BDI-IA) de 1979 (Beck, Rush, Shaw y Emery, 1979). En 1997, Vázquez y Sanz validaron el cuestionario en población española, siendo el índice de consistencia interna de 0.83 (Vázquez y Sanz, 1997; Sanz y Vázquez, 1998); la fiabilidad test-retest oscila entre 0.60 y 0.72 (Sanz y Vázquez, 1998). Cada ítem presenta cuatro alternativas de respuesta que van de 0 (ausencia del síntoma) a 3 (presencia persistente del síntoma). El rango total de puntuación varía entre 0 y 63 , con cuatro posibles puntos de corte: de 0 a 9 (ausencia de síntomas de depresión), de 10 a 18 (depresión leve), de 19 a 29 (depresión moderada) y de 30 a 63 (depresión grave).

Para medir la incidencia y gravedad de los síntomas de TEPT el instrumento utilizado fue la Escala de Gravedad de Síntomas del Trastorno de Estrés Postraumático de Echeburúa et al. (Echeburúa, de Corral, Amor, Zubizarreta y Sarasua, 1997). Esta Escala posee una consistencia interna de 0.92 ( $\alpha$ de Cronbach) y su fiabilidad test-retest a las 4 semanas de 0.89. Su eficacia diagnóstica es del $95.4 \%$. La entrevista estructurada consta de 17 ítems divididos en 3 bloques: reexperimentación ( 5 ítems), evitación ( 7 ítems) e hiperactivación ( 5 ítems) con 4 alternativas de frecuencia e intensidad de los síntomas de 0 a 3 (donde 0 es "nada" y 3 es "5 o más veces por semana/mucho"). Para determinar la presencia del TEPT se debe puntuar, al menos, en un ítem de la escala de reexperimentación, tres de la escala de evitación y dos de la escala de hiperactivación. Además cada escala posee sus puntos de corte: 5,6 y 4, respectivamente. Finalmente, el rango en la escala global es de 0 a 51 y el punto de corte se sitúa en 15 .

El tipo de violencia sufrida fue evaluada mediante un cuestionario diseñado ad hoc siguiendo la clasificación realizada por García-Linares et al. (2005).

Las mujeres fueron preguntadas por su edad, nivel de educación y estado civil, variables que fueron recogidas mediante un cuestionario diseñado ad hoc. Los niveles de respuesta recogidos para la variable nivel de educación fueron: No lee ni escribe, Lee y escribe, Certificado Escolar, Graduado Escolar, Estudios secundarios, Estudios universitarios. Los niveles de respuesta para la variable estado civil fueron: Soltera sin convivencia, Soltera con convivencia, Casada, Separada/Divorciada, Viuda.

\section{Análisis de datos}

Los datos recogidos fueron analizados empleando el programa estadístico SPSS en su versión 19 y posteriores. El punto de corte para la significación estadística fue establecido en $\mathrm{p}<0.05$.

El análisis de las variables edad, depresión y TEPT se ha efectuado empleando estadísticos descriptivos de media y desviación típica. Para efectuar comparaciones entre los tres grupos se utilizó el análisis de la varianza (ANOVA) junto con la prueba post-hoc Bonferroni. En el caso de incumplimiento de los supuestos para ANOVA se utilizó el test de Welch con la 
prueba post-hoc Games-Howell.

Complementariamente, se calculó el tamaño del efecto mediante el índice eta cuadrado parcial con el objetivo de valorar la magnitud de las diferencias de medias encontradas para las variables depresión y TEPT.

El análisis de las variables correspondientes al nivel de educación, estado civil y tipo de violencia se realizó empleando el análisis de frecuencias. Se utilizaron tablas de contingencia con la prueba $\chi^{2}$ de Pearson con el fin de determinar la relación existente entre dos variables categóricas.

\section{Resultados}

Las medias de las puntuaciones de depresión y TEPT, evaluados mediante el BDI y la Escala de Gravedad de Síntomas del Trastorno por Estrés Postraumático respectivamente, y que han determinado la inclusión de las mujeres en cada uno de los grupos, se muestran en la Tabla 1. El punto de corte para determinar la presencia de depresión fue de 18 y el de TEPT de 15 .

Tabla 1.Puntuaciones medias de depresión (BDI) y puntuaciones medias en la escala global del TEPT para las mujeres de los grupos VP sin síntomas, VP con depresión y VP con depresión/TEPT.

\begin{tabular}{lllll}
\hline Grupo & $\begin{array}{l}\text { VP sin } \\
\text { síntomas } \\
\mathrm{n}=30\end{array}$ & $\begin{array}{l}\text { VP Depresión } \\
\mathrm{n}=50\end{array}$ & $\begin{array}{l}\text { VP Depresión/TEPT } \\
\mathrm{n}=28\end{array}$ & Estadísticos \\
\hline Variable & & & & \\
\hline & & & & $\mathrm{Vw}(2,53.71)=94.82 ;$ \\
BDI & $4.63( \pm 2.7)$ & $17.76( \pm 7.47)^{* * * *}$ & $25.03( \pm 11.55)^{* * * / a \mathrm{aa}}$ & $\mathrm{p}<0.001$ \\
\hline $\begin{array}{l}\text { Puntuación } \\
\text { total }\end{array}$ & & & & $\mathrm{Vw}(2,57.91)=110.01 ;$ \\
TEPT & $4.27( \pm 4.07)$ & $10.84( \pm 5.28)^{* * *}$ & $28.61( \pm 7.74)^{* * * / \text { aaa }}$ & $\mathrm{p}<0.001$ \\
\hline
\end{tabular}

$* * * \mathrm{p}<0.001$; Difiere del grupo VP sin síntomas

aaa $\mathrm{p}<0.001 ;{ }^{\mathrm{a} a} \mathrm{p}<0.01$; Difiere del grupo VP depresión

Se observan diferencias significativas entre los grupos en cuanto a la media de la puntuación total obtenida en el BDI $\left[\mathrm{Vw}(2,53.71)=94.82 ; \mathrm{p}<0.001 ; \eta^{2}=0.5\right]$, resultando que el $50 \%$ de las diferencias encontradas se atribuyen al efecto de dicha puntuación. Las pruebas post hoc aplicadas muestran que las medias de los grupos VP con depresión/TEPT y VP con depresión fueron significativamente superiores a la media del grupo VP sin síntomas $(\mathrm{p}<0.001)$ (ver Tabla 1 y Figura 1$)$.

Con respecto al TEPT también se han observado diferencias entre las medias [ $\mathrm{Vw}(2$, $\left.57.91)=110.01 ; \mathrm{p}<0.001 ; \eta^{2}=0.7\right]$, diferencias que poseen un tamaño de efecto de alta mag- 
nitud. Las medias de los grupos VP con depresión/TEPT y VP con depresión han sido significativamente superiores a la del grupo VP sin síntomas $(\mathrm{p}<0.001)$ (ver Tabla 1 y Figura1).

Figura 1. Puntuaciones medias de depresión y TEPT para las mujeres de los grupos VP sin síntomas, VP con depresión y VP con depresión/TEPT.

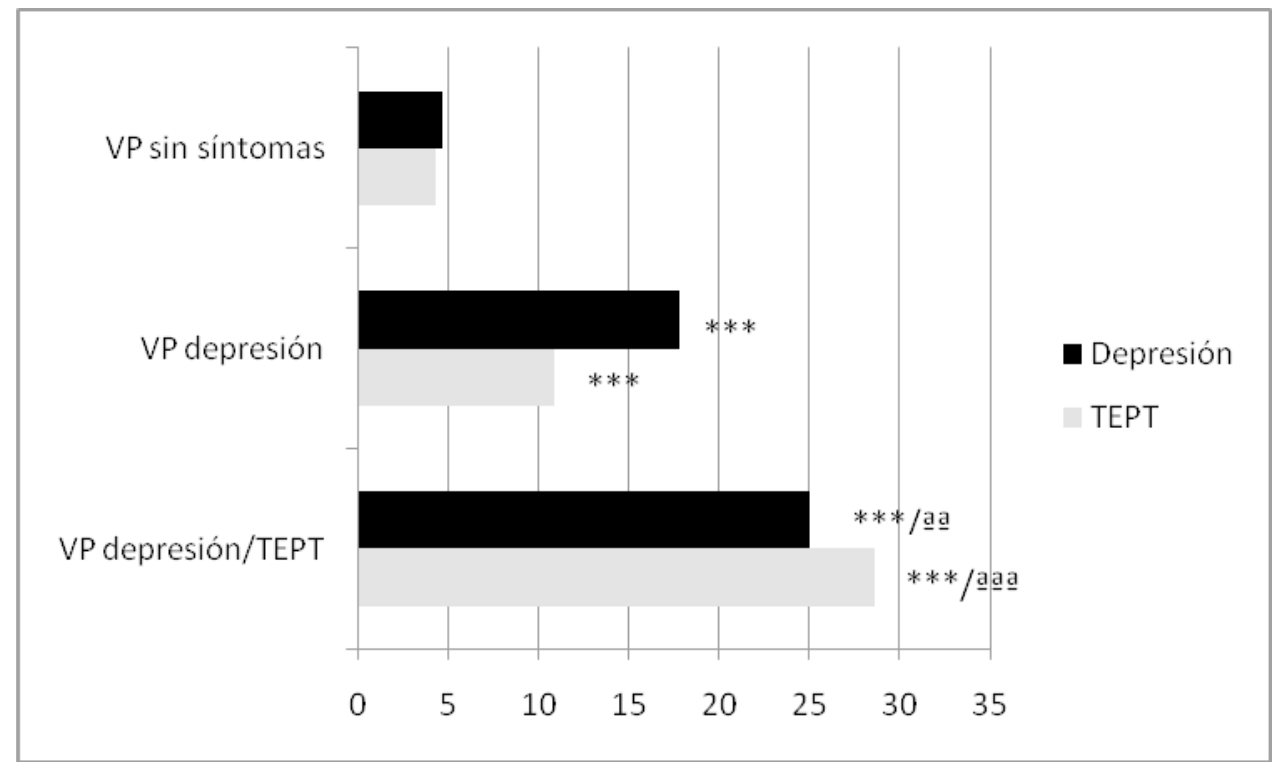

*** $\mathrm{p}<0.001$; Difiere del grupo VP sin síntomas

${ }^{\text {aaa }} \mathrm{p}<0.001 ;{ }^{\text {aa }} \mathrm{p}<0.05$; Difiere del grupo VP depresión

En cuanto a la edad de las mujeres, ésta se situó en un rango entre 24 y 68 años, presentando una edad media de 44.76 (DT=10.26). No se han producido diferencias significativas entre las medias de las edades de los grupos ( $\mathrm{p}=0.44)$ (Ver Tabla 2).

En relación con la variable nivel de educación, las mujeres de la muestra se encuentran distribuidas homogéneamente en casi todos los niveles educativos, no encontrándose una relación en cuanto al nivel de educación que poseen y la gravedad de los síntomas clínicos que presentan ( $\mathrm{p}=0.77)$ (Ver Tabla 2).

El estado civil de las mujeres tampoco estuvo asociado con la presencia/ausencia de sintomatología depresiva y de TEPT ( $\mathrm{p}=0.23$ ) (Ver Tabla 2).

Por último, los datos obtenidos en relación con el tipo de violencia sufrida reflejan que las mujeres están distribuidas de una manera homogénea entre los tres grupos, no produciéndose ninguna asociación significativa entre el tipo de violencia sufrida y el desarrollo o no de sintomatología depresiva y de TEPT. En la Tabla 3 se expone el porcentaje de mujeres que ha sido víctima de cada tipo de violencia junto con los resultados de las pruebas estadísticas aplicadas. 
Tabla 2. Datos demográficos de las mujeres de los grupos VP sin síntomas, VP con depresión y VP con depresión/TEPT y su significación estadística.

\begin{tabular}{|c|c|c|c|c|}
\hline Grupo & $\begin{array}{l}\text { VP sin sínto- } \\
\text { mas }\end{array}$ & $\begin{array}{l}\text { VP con depre- } \\
\text { sión }\end{array}$ & $\begin{array}{l}\text { VP con } \\
\text { depresión/ } \\
\text { TEPT }\end{array}$ & Estadísticos \\
\hline & $n=30$ & $\mathrm{n}=50$ & $\mathrm{n}=28$ & \\
\hline Edad & $46.43( \pm 10.22)$ & $43.42( \pm 11.03)$ & $45.4( \pm 8.8)$ & $\begin{array}{l}\text { Ww }(2,63.42) \\
=0.82 ; \text { n.s. }\end{array}$ \\
\hline Nivel de educación & & & & $\begin{array}{l}\chi^{2}(10, N=108)= \\
6.55 ; \text { n.s. }\end{array}$ \\
\hline No lee ni escribe & 0 & 2 & 3.6 & \\
\hline Lee y escribe & 10 & 14 & 3.6 & \\
\hline Certificado escolar & 23.3 & 16 & 32.1 & \\
\hline Graduado escolar & 30 & 38 & 35.7 & \\
\hline Estudios secundarios & 30 & 22 & 21.4 & \\
\hline Estudios universitarios & 6.7 & 8 & 3.6 & \\
\hline Estado civil & & & & $\begin{array}{l}\chi^{2}(8, N=108)= \\
9.97 ; \text { n.s. }\end{array}$ \\
\hline Soltera sin convivencia & 10 & 6 & 10.7 & \\
\hline $\begin{array}{l}\text { Soltera con conviven- } \\
\text { cia }\end{array}$ & 0 & 4 & 3.6 & \\
\hline Casada & 36.7 & 54 & 57.1 & \\
\hline Separada/divorciada & 43.3 & 36 & 21.4 & \\
\hline Viuda & 10 & 0 & 7.1 & \\
\hline
\end{tabular}


Tabla 3. Porcentaje de mujeres que ha sufrido cada tipo de violencia en los grupos VP sin síntomas, VP con depresión y VP con depresión/TEPT y su significación estadística.

\begin{tabular}{lllll}
\hline Grupo & $\begin{array}{l}\text { VP sin } \\
\text { síntomas }\end{array}$ & $\begin{array}{l}\text { VP con } \\
\text { depresión }\end{array}$ & $\begin{array}{l}\text { VP con depresión/ } \\
\text { TEPT }\end{array}$ & Estadísticos \\
\hline Violencia física & 56.7 & 64 & 53.6 & $\chi^{2}(2, \mathrm{~N}=108)$ \\
\hline & & $\mathrm{n}=50$ & $\mathrm{n}=28$ & $0.92 ; \mathrm{n} . \mathrm{s}$. \\
\hline $\begin{array}{l}\text { Violencia psicológica } \\
\text { Violencia sexual }\end{array}$ & 43.3 & 36 & 46.4 & $0.92 ; \mathrm{n} . \mathrm{s}$. \\
& 20 & 26 & 25 & $0.39 ; \mathrm{n} . \mathrm{s}$. \\
\hline $\begin{array}{l}\text { Violencia física+sexual } \\
\text { Violencia }\end{array}$ & 13.3 & 20 & 21.4 & $0.76 ; \mathrm{n} . \mathrm{s}$. \\
\hline
\end{tabular}

\section{Discusión}

El principal resultado encontrado en este trabajo ha sido que las mujeres víctimas de violencia de pareja pueden presentar distintos perfiles de deterioro en su salud mental en cuanto a la manifestación de sintomatología de depresión y TEPT se refiere. La evaluación de dichos perfiles en relación con el tipo de violencia sufrida ha evidenciado que no es esta variable la que determina dichas diferencias individuales, habiendo sido controladas las características demográficas edad, nivel de educación y estado civil.

Investigaciones previas han establecido que variables como el tipo de violencia (física, psicológica, sexual) y la frecuencia, duración o gravedad de la misma, determinan el desarrollo de psicopatología en las mujeres víctimas de violencia de pareja (Becker, Stuewig y McCloskey, 2010; Bennice et al., 2003; Blasco-Ros, Sánchez-Lorente y Martínez, 2010; Coker, Weston, Creson, Justice y Blakeney, 2005; Devries et al., 2013; MacHisa, Christofides y Jewkes, 2017; Martínez-Torteya, Bogat, Von Eye, Levendosky y Davidson, 2009; Nathanson, Shorey, Tirone y Rhatigan, 2012; Picó-Alfonso, 2005; Wathen et al., 2016). Señalar como ejemplo que Bennice et al. (2003) encontraron que la violencia sexual estaba relacionada con la presencia del TEPT, mientras que Picó-Alfonso (2005) encontró que era la violencia psicológica la que actuaba como un importante predictor del TEPT.

Sin embargo, como se ha comentado anteriormente, en el presente trabajo no han sido halladas relaciones significativas entre la incidencia de sintomatología depresiva y de TEPT y el tipo de violencia (física, psicológica y/o sexual) que han sufrido las mujeres, resultado 
que, no obstante, también había sido encontrado en otros trabajos previos que valoraron la relación entre el tipo, duración o gravedad de la violencia y la gravedad de la sintomatología presentada por las mujeres (Hellmuth et al., 2014; Johnson y Zlotnick, 2012; PatróHernández et al., 2007).

Por tanto, estos datos indicarían que las mujeres víctimas de violencia de pareja no conforman un grupo homogéneo entre ellas, al mostrar distintos perfiles de deterioro en su salud mental aun habiendo sido víctimas de las mismas situaciones de violencia. Consecuentemente se obtiene que no debiera utilizarse el deterioro presentado por las mujeres en su salud mental para valorar la gravedad de la violencia, puesto que más bien habría que considerar que pudiera tratarse de variables independientes.

Estudios realizados desde la psicología positiva evidencian que casi en el 50\% de los casos la respuesta emitida ante una experiencia traumática es la resiliencia, seguida de la recuperación espontánea, en un 30\% de los casos (Muñoz, 2013). Asimismo, la evaluación de estas variables en un estudio longitudinal (Blasco-Ros et al., 2010) estableció que con el paso del tiempo las mujeres que habían sufrido violencia física y psicológica consiguieron recuperar su estatus mental, no así las mujeres que sólo habían sufrido violencia psicológica, lo cual muestra de nuevo las diferencias individuales existentes entre este colectivo.

Lo hasta aquí expuesto posee gran relevancia para el ámbito legal, ya que a nivel judicial suele esperarse que las mujeres tengan un perfil único de deterioro de salud mental (delitosecuela). No obstante, en la evaluación forense de las víctimas se observa frecuentemente cómo los factores de vulnerabilidad o de protección actúan como potentes variables mediadoras en la evolución del daño psicológico que manifiestan las mujeres. Por ello, tal y como establece Muñoz (2013), no debe confundirse la utilización de la valoración del daño psíquico para fundamentar hechos probados, resultando igual de relevante que no se asocie la ausencia de deterioro en la salud mental, por una adecuada resiliencia de la víctima, con una menor gravedad de la violencia sufrida.

Así, de los resultados concretos de este trabajo podemos señalar que frente a la vivencia de las mismas situaciones de violencia, unas mujeres se mantienen asintomáticas, otras presentan sintomatología depresiva y otras desarrollan sintomatología de mayor gravedad (depresión en comorbilidad con un TEPT), debiendo ser, por tanto, otros factores distintos a la violencia sufrida, los que estén determinando las diferencias individuales existentes entre las mujeres (Savall-Rodríguez, 2015). La introducción, en estudios posteriores, de nuevas variables permitirá realizar análisis estadísticos más concretos (análisis de moderación, por ejemplo), permitiendo examinar cuáles son las variables que puedan estar mediando en estos resultados.

Por tanto, se considera relevante la realización de un mayor número de trabajos en este sentido, ya que, de generalizarse estos resultados, resultaría de gran importancia su visualización a los operadores judiciales, a fin de que a la hora de enjuiciar un delito de estas características se tenga en consideración que aunque no haya deterioro puede haber víctima.

Por último, señalar como una limitación de este estudio el no contar con un grupo de mujeres que únicamente presentara TEPT, ya que es numerosa la bibliografía que establece sus 
conclusiones en base a este trastorno (uno de los más frecuentes en víctimas de violencia). Por ello, hubiera resultado muy interesante comprobar si las mujeres que sólo presentan TEPT han experimentado situaciones de violencia diferentes de las mujeres que sólo presentan depresión o de las que presentan depresión/TEPT.

\section{Referencias}

Arce, R.; Fariña, F. (2009). Evaluación psicológica forense de la credibilidad y daño psíquico en casos de violencia de género mediante el Sistema de Evaluación Global. En F. Fariña, R. Arce y G. Buela-Casal (Eds.), Violencia de género. Tratado psicológico y Legal (pp. 147-168). Madrid: Biblioteca Nueva.

Beck, A.T.; Rush, A.J.; Shaw, B.F.; Emery, G. (1979). Cognitive therapy of depression. New York: Guilford Press.

Becker, K.D.; Stuewig, J.; McCloskey, L.A. (2010). Traumatic stress symptoms of women exposed to different forms of childhood victimization and intimate partner violence. Journal of Interpersonal Violence, 25(9), 16991715. DOI: $10.1177 / 0886260509354578$.

Bennice, J.A.; Resick, P.A.; Mechanic, M.; Astin, M. (2003). The relative effects of intimate partner physical and sexual violence on post-traumatic stress disorder symptomatology. Violence and Victims, 18(1), 87-94.

Blasco-Ros, C.; Sánchez-Lorente, S.; Martínez, M. (2010). Recovery from depressive symptoms, state anxiety and posttraumatic stress disorder in women exposed to physical and psychological, but not to psychological intimate partner violence alone: a longitudinal study. BMC Psychiatry, 10:98. DOI: 10.1186/1471-244X-10-98.

Campbell, J. C. (2002). Health consequences of intimate partner violence. Lancet, 359(9314), 1331-1336.

Chuang, C.H.; Cattoi, A.L.; McCall-Hosenfeld, J.S.; Camacho, F.; Dyer, A.; Weisman, C.S. (2012). Longitudinal association of intimate partner violence and depressive symptoms. Mental Health in Family Medicine, 9(2), 107-114.

Coker, A.L.; Weston, R.; Creson, D.L.; Justice, B.; Blakeney, P. (2005). PTSD symptoms among men and women survivors of intimate partner violence: The role of risk and protective factors. Violence and Victims, 20(6), 625-643.

Craparo, G.; Gori, A.; Petruccelli, I.; Cannella, V.; Simonelli, C. (2014). Intimate partner violence: Relationships between alexithymia, depression, attachment styles, and coping strategies of battered women. Journal of Sexual Medicine, 11(6), 1484-1494. DOI: 10.1111/jsm.12505.

Devries, K.M.; Mak, J.Y.; Bacchus, L.J.; Child, J.C.; Falder, G.; Petzold, M. et al. (2013). Intimate partner violence and incident depressive symptoms and suicide attempts: A systematic review of longitudinal studies. PLoS Medicine, 10(5). D0I: 10.1371/journal.pmed.1001439.

Dougé, N.; Lehman, E. B.; McCall-Hosenfeld, J.S. (2014). Social support and employment status modify the effect of intimate partner violence on depression symptom severity in women: Results from the 2006 behavioral risk factor surveillance system survey. Women's Health Issues, 24(4), 425-434. DOI: 10.1016/j.whi.2014.03.006.

Dutton, M.A.; Green, B.L., Kaltman, S.I.; Roesch, D.M.; Zeffiro, T.A.; Krause, E.D. (2006). Intimate partner violence, PTSD, and adverse health outcomes. Journal of Interpersonal Violence, 21(7), 955-968. D0I: $10.1177 / 0886260506289178$.

Echeburúa, E.; de Corral, P.; Amor, P.J.; Zubizarreta, I.; Sarasua, B. (1997). Escala de gravedad de Síntomas de Trastorno de Estrés Postraumático: propiedades psicométricas. Análisis y Modificación de Conducta, 23(90), 503526.

Ehring, T., Ehlers, A., Cleare, A. J.; Glucksman, E. (2008). Do acute psychological and psychobiological responses to trauma predict subsequent symptom severities of PTSD and depression? Psychiatry Research, 161(1), 67-75. DOI: 10.1016/j.psychres.2007.08.014

García-Linares, M.I.; Picó-Alfonso, M.A.; Sánchez-Lorente, S.; Savall-Rodríguez, F.; Celda-Navarro, N.; Blasco-Ros, C.; Martínez, M. (2005). Assessing physical, sexual, and psychological violence perpetrated by intimate male partners toward women: a Spanish cross-sectional study. Violence and Victims, 20(1), 99-123. 
Griffin, M.G.; Resick, P.A.; Yehuda, R. (2005). Enhanced cortisol suppression following dexamethasone administration in domestic violence survivors. American Journal of Psychiatry, 162(6), 1192-1199. D0I: 10.1176/appi. ajp.162.6.1192.

Hansen, N.B.; Eriksen, S.B.; Elklit, A. (2014). Effects of an intervention program for female victims of intimate partner violence on psychological symptoms and perceived social support. European Journal of Psychotraumatology, 5, 10.3402/ejpt.v5.24797. eCollection 2014. DOI: 10.3402/ejpt.v5.24797.

Hardeveld, F.; Spijker, J.; Vreeburg, S. A.; De Graaf, R.; Hendriks, S. M.; Licht, C. M. M. et al. (2014). Increased cortisol awakening response was associated with time to recurrence of major depressive disorder. Psychoneuroendocrinology, 50, 62-71. DOI: 10.1016/j.psyneuen.2014.07.027

Heim, C., Newport, D. J., Heit, S., Graham, Y. P., Wilcox, M., Bonsall, R. et al. (2000). Pituitary-adrenal and automatic responses to stress in women after sexual and physical abuse in childhood. Journal of the American Medical Association, 284(5), 592-597.

Hellmuth, J.C.; Jaquier, V.; Swan, S.C.; Sullivan, T.P. (2014). Elucidating posttraumatic stress symptom profiles and their correlates among women experiencing bidirectional intimate partner violence. Journal of Clinical Psychology, 70(10), 1008-1021. DOI: 10.1002/jclp.22100.

Honda, T.; Wynter, K.; Yokota, J.; Tran, T.; Ujiie, Y.; Niwa, M. et al. (2017). Sexual Violence as a Key Contributor to Poor Mental Health Among Japanese Women Subjected to Intimate Partner Violence. Journal of Women's Health. Sep 7. https://doi.org/10.1089/jwh.2016.6276

Johnson, D.M.; Zlotnick, C. (2012). Remission of PTSD after victims of intimate partner violence leave a shelter. Journal of Traumatic Stress, 25(2), 203-206. DOI: 10.1002/jts.21673.

Johnson, D.M.; Delahanty, D.L.; Pinna, K. (2008). The cortisol awakening response as a function of PTSD severity and abuse chronicity in sheltered battered women. Journal of Anxiety Disorders, 22(5), 793-800. D0I: 10.1016/j.janxdis.2007.08.00

Kaysen, D.; Resick, P.A.; Wise, D. (2003). Living in danger: The impact of chronic traumatization and the traumatic context on posttraumatic stress disorder. Trauma, Violence \& Abuse, 4(3), 247-264. D0I: $10.1177 / 1524838003004003004$.

Kelly, U.A.; Pich, K. (2014). Community-based PTSD treatment for ethnically diverse women who experienced intimate partner violence: A feasibility study. Issues in Mental Health Nursing, 35(12), 906-913. D0I: 10.3109/01612840.2014.931496.

Koolhaas, J.M.; Bartolomucci, A.; Buwalda, B.; de Boer, S.F.; Flügge, G.; Korte, S.M. et al. (2011). Stress revisited: A critical evaluation of the stress concept. Neuroscience and Biobehavioral Reviews, 35(5), 1291-1301. D0I: 10.1016/j.neubiorev.2011.02.003.

Ley 4/2015, de 27 de abril, del Estatuto de la víctima del delito. B.0.E. núm. 101, de 28 de abril de 2015.

Lilly, M.M.; Howell, K.H.; Graham-Bermann, S. (2014). World assumptions, religiosity, and PTSD in survivors of intimate partner violence. Violence Against Women, 21(1), 87-104. DOI: 10.1177/1077801214564139.

Lok, A., Mocking, R. J. T., Ruhé, H. G., Visser, I., Koeter, M. W. J., Assies, J. et al. (2012). Longitudinal hypothalamicpituitary-adrenal axis trait and state effects in recurrent depression. Psychoneuroendocrinology, 37(7), 892-902. DOI: 10.1016/j.psyneuen.2011.10.005

MacHisa, M.T.; Christofides, N.; Jewkes, R. (2017). Mental ill health in structural pathways to women's experiences of intimate partner violence. PLOS ONE, 12 (4): e0175240. DOI: 10.1371/journal.pone.0175240.

Martínez-Torteya, C.; Bogat, G.A.; Von Eye, A.; Levendosky, A.A.; Davidson, W.S. (2009). Women's appraisals of intimate partner violence stressfulness and their relationship to depressive and posttraumatic stress disorder symptoms. Violence and Victims, 24(6), 707-722.

McEwen, B. S. (2004). Protection and damage from acute and chronic stress: Allostasis and allostatic overload and relevance to the pathophysiology of psychiatric disorders. Annals of the New York Academy of Sciences, 1032, 1-7. DOI: 10.1196/annals. 1314.001

Muñoz, J.M. (2013). La evaluación psicológica forense del daño psíquico: propuesta de un protocolo de actuación pericial. Anuario de Psicología Jurídica, 23, 61-69. 
Nathanson, A.M.; Shorey, R.C.; Tirone, V.; Rhatigan, D.L. (2012). The prevalence of mental health disorders in a community sample of female victims of intimate partner violence. Partner Abuse, 3(1), 59-75. D0I: 10.1891/19466560.3.1.59.

Organización Mundial de la Salud (2006). Violencia de pareja y violencia sexual contra la mujer. Disponible en: http:// www.who.int/mediacentre/factsheets/fs239/es/

Patró-Hernández, R.; Corbalán, F.J.; Limiñana, R.M. (2007). Depresión en mujeres maltratadas: Relaciones con estilos de personalidad, variables contextuales y de la situación de violencia. Anales de Psicología, 23(1), 118-124.

Pérez, S.; Johnson, D.M. (2008). PTSD compromises battered women's future safety. Journal of Interpersonal Violence, 23(5), 635-651. DOI: 10.1177/0886260507313528.

Pico-Alfonso, M.A. (2005). Psychological intimate partner violence: The major predictor of posttraumatic stress disorder in abused women. Neuroscience and Biobehavioral Reviews, 29, 181-193. D0I: 10.1016/j.neubiorev.2004.08.010.

Sanz, J. y Vázquez, C. (1998). Fiabilidad, validez y datos normativos del inventario de depresión de Beck. Psicothema, 10(2), 303-318.

Sarasua, B.; Zubizarreta, I.; Echeburúa, E.; De Corral, P. (2007). Psychopathological profile of battered women according to age. [Perfil psicopatológico diferencial de las víctimas de violencia de pareja en función de la edad] Psicothema, 19(3), 459-466.

Savall-Rodríguez, F. (2015). Variables biopsicosociales que contribuyen al diferente impacto de la violencia de pareja en la salud de las mujeres: factores de vulnerabilidad y resiliencia. (Tesis Doctoral). Valencia: Universitat de València.

Torres, A.; Garcia-Esteve, L.; Navarro, P.; Tarragona, M.J.; Imaz, M.L.; Ascaso, C. et al. (2013). Relationship between intimate partner violence, depressive symptomatology, and personality traits. Journal of Family Violence, 28(4), 369-379.

Vázquez, C. y Sanz, J. (1997). Fiabilidad y valores normativos de la versión española del Inventario para la Depresión de Beck de 1978. Clínica y Salud, 8(3), 403-422.

Wathen, C.N.; Tanaka, M.; MacGregor, J.C.D.; Ferro, M.A.; McKee, C.; Boyle, M.; Ford-Gilboe, M.; MacMillan, H.L. (2016) Trajectories for women who disclose intimate partner violence in health care settings: the key role of abuse severity. International Journal of Public Health, 61(8), 873-882. D0I: 10.1007/s00038-016-0852- 